\section{SCIENCE CHINA \\ Physics, Mechanics \& Astronomy}

- Editorial •

Editor's Focus
June 2021 Vol. 64 No. 6: 267261

https://doi.org/10.1007/s11433-021-1660-3

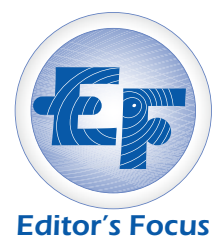

\title{
A new type of Andreev reflection
}

Andreev reflection (AR) [1] refers to a peculiar quasiparticle reflection process that takes place at the interface between a normal metal and a superconductor. Specifically, an electron impinging from the normal metal is reflected back as a hole, which effectively describes that two electrons in the normal metal penetrate into the superconductor and form a Cooper pair. AR dominates the electron transport below the superconducting gap in the superconductor junctions, which is an important experimental approach for the detection of various properties of electronic systems, such as the pairing symmetry of the unconventional superconductors, the spin polarization of the itinerant electrons and most recently, Majorana zero mode in topological systems.

In the conventional AR or so-called retro AR, the reflected hole retraces the path of the incident electron by inverting all velocity components [1]. Later, Beenakker [2] proposed that specular AR can occur in graphene as the electron and hole are from the conduction and valence band, respectively, in which only the velocity component perpendicular to the interface is inverted. In two dimensions, the two types of AR constitute a complete set of AR, while the story gets enriched in a three-dimensional junction. In a recent study, Luo et al. [3] from Nanjing University proposed the third type of AR, dubbed "anomalous Andreev reflection", in which two of three velocity components are inverted, giving rise to a peculiar reflection trajectory in real space. They showed that such an anomalous AR can be realized in a class of materials carrying a torus-shaped Fermi surface, such as the doped nodal line semimetals [4-6]. The Fermi torus doubles the reflection channels when its toroidal circle is perpendicular to the interface of the junction, which generates multiple AR processes. In particular, the anomalous AR dominates in the light doping regime and can be visibly manifested as a unique ridge structure of the spatially resolved nonlocal conductance spectra.

The present work opens a new research avenue for the AR spectroscopy and offers a clear transport signature of the peculiar torus-shaped Fermi surface. Moreover, such an anomalous reflection trajectory has not been discovered in any physical system, neither in condensed matter nor in optics. Therefore, it may also stimulate explorations in other physical systems, such as optics and acoustics.

XinCheng Xie

School of Physics, Peking University, Beijing 100871, China

1 A. F. Andreev, Sov. Phys. JETP 19, 1228 (1964).

2 C. W. J. Beenakker, Phys. Rev. Lett. 97, 067007 (2006), arXiv: condmat/0604594.

3 W. Luo, W. Chen, and D. Xing, Sci. China-Phys. Mech. Astron. 64, $267262(2021)$

4 A. A. Burkov, M. D. Hook, and L. Balents, Phys. Rev. B 84, 235126
(2011), arXiv: 1110.1089.

5 C. Fang, H. Weng, X. Dai, and Z. Fang, Chin. Phys. B 25, 117106 (2016), arXiv: 1609.05414.

6 B. Chen, B. Zhang, J. H. Yu, F. C. Fei, M. Naveed, Y. Zhang, Z. Sun, X. G. Wan, and F. Q. Song, Sci. China-Phys. Mech. Astron. 63, 227011 (2020). 